\title{
Risk Categorization with Different Grades of Cervical Pre-Neoplastic Lesions - High Risk HPV Associations and Expression of p53 and RAR
}

\author{
D Ghosh ${ }^{1 *}$, A K Roy ${ }^{1}$, N Murmu ${ }^{2}$, S Mandal ${ }^{3}$, A Roy $^{4}$
}

\begin{abstract}
Objective: To identify high risk HPV associations by evaluating linked $\mathrm{p} 16$ overexpression and also the expression of $\mathrm{p} 53$ and RAR $\beta$ together with histopathology for risk categorization of cervical pre-neoplastic lesions. Materials and Methods: Immunohistochemical staining was performed on 100 cases of cervical pre- neoplastic lesions for expression of biomarkers like p16, p53 and RAR $\beta$ for comparison with haematoxylin/eosin (HE) findings. All the experimentally generated data were statistically analyzed. Results: In this study $70 \%$ cases showed overexpression of p16INK4A increasing progressively from CIN I to CIN II but reduced in CIN III $(\mathrm{p}<0.01)$. p53 oncoprotein expression was seen in $51 \%$ cases, again with increments from CIN I to CIN II with slight reduction in CIN III ( $p<0.01$ ). Some $24 \%$ cases showed negative immunoreactivity for the putative tumor suppressor gene RAR $\beta$ ( $p>0.05$ ). Conclusion: Our study provides support for the idea that p16 can be used to identify associations with HPV, as well as having potential along with p53 and RAR $\beta$ for categorizing cervical pre-neoplastic cases having a higher risk of neoplastic conversion. Thus it may be concluded that accurate risk categorization can be achieved with the help of genetic markers as well as histopathology.
\end{abstract}

Keywords: Cervical cancer- histopathology- immunohistochemistry- papilloma viruses- tumour markers

Asian Pac J Cancer Prev, 20 (2), 549-555

\section{Introduction}

Worldwide, cervical cancer is the fourth most common cancer and the second leading cause of death in women aged 15-44 years, with an estimated 569,847 new cases and 311,365 deaths in 2018. In India, cervical cancer is the second most common cancer in women with 96,922 new cases and 60,078 deaths in 2018 (Bray et al., 2018). Organised screening programmes are generally considered the milestone in cervical cancer prevention.

Cervical cancer is preceded by pre-neoplastic lesions, cervical intraepithelial neoplasia (CIN) and histopathologically, it is classified as mild (CIN I), moderate (CIN II) and severe (CIN III). Approximately 1.5 per 1000 women in developed countries is diagnosed with CIN II/CIN III annually (Tainio et al., 2018).

Human papilloma viruses (HPVs) are the main etiological factor in the occurrence of pre-neoplastic lesions and cervical cancer. High Risk-HPVs (HR-HPVs) are the main causal agent of cervical cancer and are present in $95 \%$ of cervical infections (Udar and Rader, 2014). In India, HR-HPV 16 and 18 are the most common HPVs and are responsible for $82.7 \%$ of invasive carcinoma
(Mishra et al., 2016). The majority of HPV infections regress spontaneously without treatment, only small percentage of cases infected with persistent HR-HPV develop pre-neoplastic cervical lesions (Hwang et al., 2012) of which very few develop invasive cancer unless detected and treated (Udar and Rader, 2014; Guitrezz et al., 2015). In addition, it has been seen that many cases of CIN III remained stable for many years whereas patients with CIN I carried significant risk and progressed to malignancy within short period of time (Kim et al., 2001). This accentuates the importance of risk categorization diagnosis as well as identification of those pre-neoplastic lesions that are at highest risk of progression.

Histopathological examination is considered as the "gold standard" in the assessment of cervical lesions, however, it confers little or no information regarding the risk of persistence, progression or regression of cancer (Hwang et al., 2012; Wu et al., 2014). HPV infections and molecular events supersede cell cycle controls, the immune detection of cell proteins that are differentially expressed in infected cells is currently being considered for use as potential tumour and prognostic marker, to improve diagnostic accuracy as well as to identify those

${ }^{1}$ Department of Pathology and Cancer Screening, ${ }^{2}$ Department of Signal Transduction and Biogenic Amines, ${ }^{3}$ Department of Epidemiology and Biostatistics, Chittaranjan National Cancer Institute, ${ }^{4}$ Institute of Medicine and Sagar Dutta Hospital, Kolkata, India.*For Correspondence: dipanwitaghosh88@gmail.com 
patients at risk for progression to cancer (Sarma et al., 2017). Under such perspective, biomarker study in combination with histopathology increases the sensitivity.

HPV integration into the host genome is a critical step in the process of cervical carcinogenesis and cervical cancer which leads to increase in the expression of E6 and E7 viral oncoproteins that have the ability to inactivate p53 and retinoblastoma protein ( $\mathrm{pRb}$ ) respectively (Uyar and Rader, 2014). Inactivation of $\mathrm{Rb}$ protein leads to overexpression of p16INK4a thus it is considered as a surrogate marker for HR-HPV associated lesions and can discriminate integrated from non integrated HPV infection (Sarma et al., 2017; Lesnikova et al., 2009). p53 can be functionally inactivated in cervical carcinoma either by association with E6 or mutation in the gene (Stiasny et al., 2017). Due to these p53 abnormalities, cervical epithelial cell is unable to exit the cell cycle leading to genetic instability and are responsible for the development of cervical cancer (Godoy et al., 2014; Raju et al., 2015). Immunohistochemically, detection of wild type $\mathrm{p} 53$ is difficult due to very short half life but IHC can detect mutated $\mathrm{p} 53$ protein or oncoprotein thus rendering valuable prognostic information and can be useful for risk categorization.

RAR $\beta$ (Retinoic Acid Receptor Beta) is a putative tumour suppressor gene and a member of nuclear receptor RAR (Retinoic Acid Receptor). It is a negative regulator of viral oncogenes E6 and E7 thus decreased expression of RAR $\beta$ may be an important step towards malignant progression of HPV-positive cells (Ivanova et al., 2002, Wongwarankana et al., 2018). Therefore it can be used as a useful biomarker to identify the cases that are at higher risk of cancer conversion and is a striking feature in human carcinomas including head and neck, breast, oral, pancreas and carcinoma of uterine cervix (Geisen et al., 2000).

In the present study, we aimed to evaluate high grade HPV association by $\mathrm{p} 16$, expression of $\mathrm{p} 53$ and RAR $\beta$ in pre-neoplastic cervical lesions for risk categorization.

\section{Materials and Methods}

In this study a total of 100 cervical pre-neoplastic cases including 9 associated lesions were selected from patients attending CDC OPD (Cancer Detection Centre Out Patient Department) of Department of Pathology and Cancer Screening at Chittaranjan National Cancer Institute, Kolkata, during the period of 2014 to 2017 and punch biopsy was collected for further analysis at the Department of Pathology and Cancer Screening. Written informed consent was obtained from all patients and the study was approved by Institutional Ethical Committee. Biopsy specimen was fixed in 10\% formalin and processed within 24 hours. After the routine processing, paraffin tissue blocks were made and cut on a microtome in serial sections and deparaffinised sections were then stained with haematoxylin and eosin (HE). The slides were reviewed and graded according to the criteria of the World Health Organisation as CIN I, CIN II, CIN III and other associated cervical lesions (Harsh, 2010) by distinguished pathologist.

\section{Immunohistochemistry}

For immunohistochemistry of p16, p53 and RAR $\beta$ protein expression, IHC was performed on deparaffinised sections and positive controls according to the protocol of IHC World (http://www.ihcworld.com/protocol_database. html) with slight modification and commercially available kit (IHC Select- HRP/DAB Kit Millipore) was used for detection. Briefly, antigen retrieval was carried out by heating the slides in $0.01 \mathrm{M}$ citrate buffer, $\mathrm{pH} 6.0$ in microwave oven for 10 minutes. Endogenous peroxidase was blocked by $3 \%$ hydrogen peroxide in water for 10 minutes. Non specific binding was blocked by blocking reagent for 5 minutes and slides were not washed down. Monoclonal antibodies against p16 (clone 2D9A12; 1:600; abcam), p53 (clone BP53-12; 1:400; Sigma), and RAR $\beta$ (clone EPR2017, 1:50; abcam) were applied and incubated for 20 minutes at $25^{\circ} \mathrm{C}$ for $\mathrm{p} 16$ and overnight at $4^{\circ} \mathrm{C}$ for p 53 and RAR $\beta$. Further, sections were sequentially incubated with secondary antibody for 10 minutes. Next Streptavidin HRP (Horseradish Peroxidase) was applied and incubated for 10 minutes. Chromogen DAB (3,3'- Diaminobenzidine) solution was freshly prepared and added to the tissue sections and incubated for 10 minutes and then counterstained by Meyer's haematoxylin for 1 minute. The slides were then passed through a series of graded alcohol and xylene and mounted with DPX. Negative control sections were processed by eliminating the use of respective primary antibodies. After each step slides were washed with rinse buffer thoroughly and only excess liquid around the section were blotted with tissue paper.

\section{Scoring method}

Scoring of p16 immunohistochemistry was done according to Chin Ping Han et al., (2009). In each case a total of 1,000 cells were counted at $40 \mathrm{X}$ and was scored according to the intensity of the nuclear or cytoplasmic staining (no staining-0; weak staining-1; moderate staining-2; strong staining-3) and the extent of stained cells (0\%-0; 1-10\%-1; 11-50\%-2; 51-80\%-3; 81-100\%-4). The final score was determined by multiplying the intensity and extent of positive cells ranging from 0 to 12 . Score of 4 to 12 is denoted as positive or overexpression and 0 to 3 score is denoted as negative.

The p53 and RAR $\beta$ expression were analyzed semi quantitatively by counting 1,000 cells at $40 \mathrm{X}$ under light microscope. p53 immunoreactivity was considered positive in cases having more than $10 \%$ positive nucleus (Qin et al., 2002). In case of RAR $\beta$, cells having no or less than $10 \%$ nuclear and cytoplasm positive cells were considered negative and included in the study (Chakravarti et al., 2003).

\section{Statistical Analysis}

Statistical Analysis was performed with the help of Epi Info (TM) 7.2.2.2. EPI INFO is a trademark of the Centers for Disease Control and Prevention (CDC). Descriptive statistical analyses were performed to calculate the means with corresponding standard deviations (s.d.). Test of proportion was used to find the Standard Normal Deviate (Z) to compare the difference proportions and Chi-square 
$\left(\varkappa^{2}\right)$ test was performed to find the associations. In the cases where one of the cell frequencies were less than 5 corrected Chi-square $\left(x^{2}\right)$ was used to find the association between variables. t-test was used to compare the means. $\mathrm{p}<0.05$ was taken to be statistically significant.

\section{Results}

100 cases of cervical pre-neoplastic lesions including 9 other associated cervical lesions were included in the study. The mean age of the patients was $42.21 \pm 9.08$ years with range $20-55$ years and the median age of the patients was 42 years.

Histopathologically, there were 36 cases $(36 \%)$ of CIN I, 18 cases (18\%) were CIN II, 37 cases (37\%) were CIN III (Figure 1) and 9 cases $(9 \%)$ with other pathological conditions including chronic cervicitis (2 cases), koilocytotic changes (4 cases), reactive cellular changes (RCC) (1 case) and condyloma ( 2 cases).

Overexpression of p16 was seen in 70\% (70/100) of cases and test of proportion showed that overexpression was significantly higher in CIN I and CIN II (CIN I - $72.22 \%$, CIN II - 83.33\%) but decreased in CIN III $(62.16 \%)$ (Figure 2). $66.67 \%$ cases of 9 other associated lesions 6 cases (condylomas 2, cervicitis 1, RCC 1, Koilocytosis 2) showed positivity for p16. Test of proportion showed that proportion of expression was significantly higher in all the four layers of the cervical tissue $(68.57 \%)$ followed by expression in superficial and intermediate layer $(15.71 \%)(\mathrm{p}<0.001)$. p53 positivity was seen in $51 \%(51 / 100)$ cases, which increased from CIN I to CIN II (CIN I - 47.22\%, CIN II - 61.11\%) and slightly decreased in CIN III (54.05\%) (Figure 3 ). $33.33 \%$ other associated lesions showed p53 positivity
Biomarkers Study for Risk of Cervical Cancer

(condylomas 1 , cervicitis 1, RCC 1 ). In case of RAR $\beta$, 24 cases $(24 \%)$ showed negative immunoreactivity and remaining 76 cases $(76 \%)$ showed immunopositivity (Figure 4). The test of proportion showed that RAR $\beta$ negativity was significantly higher in CIN III (32.43\%) than CIN II (22.22\%) and CIN I (16.67\%) (p<0.001). 2 cases $(22.22 \%)$ with other pathological condition showed immunonegativity for RAR $\beta$.

p16 showed significant overexpression followed by p53 which showed moderate expression in all the grades of cervical pre-neoplastic and associated lesions $(p<0.01)$ whereas RAR $\beta$ immunonegativity was statistically not significant $(\mathrm{p}>0.05)$ (Table 1) (Figure 5).

There were 6 cases $(6 \%)$ out of 100 that showed overexpression of $\mathrm{p} 16$, immunoreactivity for $\mathrm{p} 53$ and negative staining for RAR $\beta$ of which 1 case $(16.66 \%)$ was CIN I, 1 case (16.67\%) were CIN II, 3 cases $(50 \%)$ were CIN III and 1 case (16.67\%) was of other associated cervical conditions.

\section{Discussion}

Cervical cancer is the leading cause of mortality and morbidity among women in the developing countries than developed countries. Association of high risk HPVs are considered as the most important etiological factor linked to cervical neoplastic and pre-neoplastic lesions (Uyar and Rader, 2014). Routine histology is the gold standard for pathological characterization of cases for risk assessment but it has certain limitations such as many cases of cervical cancer skip the pre-invasive cervical lesions before developing malignancy (Lesnikova et al., 2009) hence biomarker study along with histopathology can be accurate in predicting the outcome of the individual

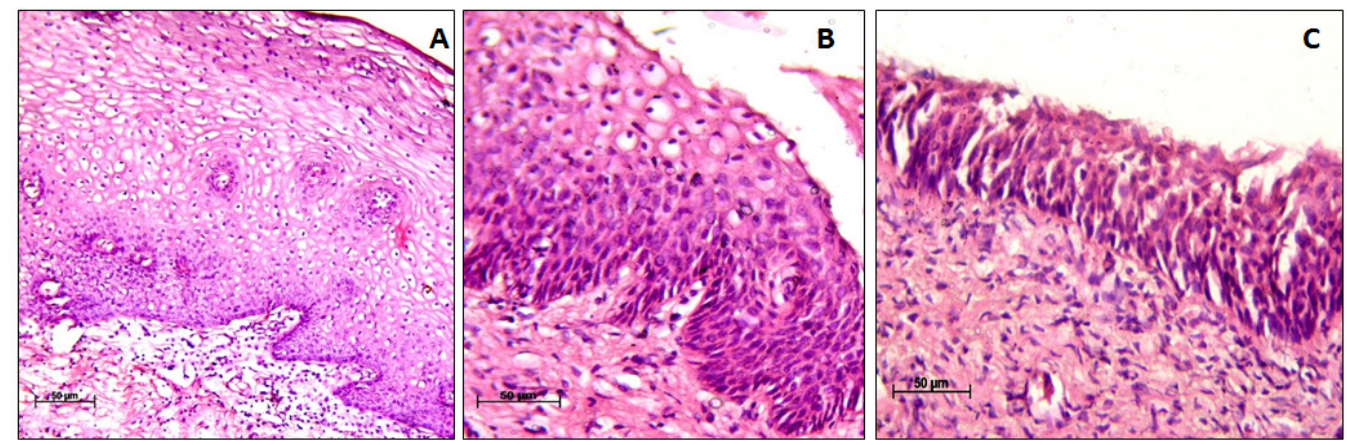

Figure 1. Haematoxylin/Eosin Staining. (A), Cervical Intraepithelial Neoplasia (CIN) I x 10X; (B), CIN II x 20X; (C), CIN III x 20X

Table 1. Immunohistochemical Expression of p16, p53 and RAR $\beta$ in Pre-Neoplastic and Other Associated Lesions of Uterine Cervix

\begin{tabular}{lccccccc}
\hline Parameters & Biomarkers & $\begin{array}{c}\text { No of cases (Total } \\
\text { cases }=100 \text { of age } \\
\text { range 20-55 yrs) }\end{array}$ & CIN I $n=36$ & CIN II $n=18$ & CIN III $n=37$ & $\begin{array}{c}\text { Other Associated } \\
\text { lesions } n=9\end{array}$ & p values \\
\hline Expression of & $\mathrm{p} 16+$ & $70(70 \%)$ & $26(72.22 \%)$ & $15(83.33 \%)$ & $23(62.16 \%)$ & $6(66.67 \%)$ & $<0.01 *$ \\
Biomarkers & $\mathrm{p} 53+$ & $51(51 \%)$ & $17(47.22 \%)$ & $11(61.11 \%)$ & $20(54.05 \%)$ & $3(33.33 \%)$ & $<0.01 *$ \\
& RAR $\beta$ & $24(24 \%)$ & $6(16.67 \%)$ & $4(22.22 \%)$ & $129(32.43 \%)$ & $2(22.22 \%)$ & $>0.05($ NS) \\
& & & & $(\mathrm{p}<0.001) * *$ & & \\
\hline
\end{tabular}

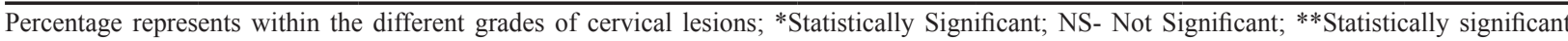
within the group 


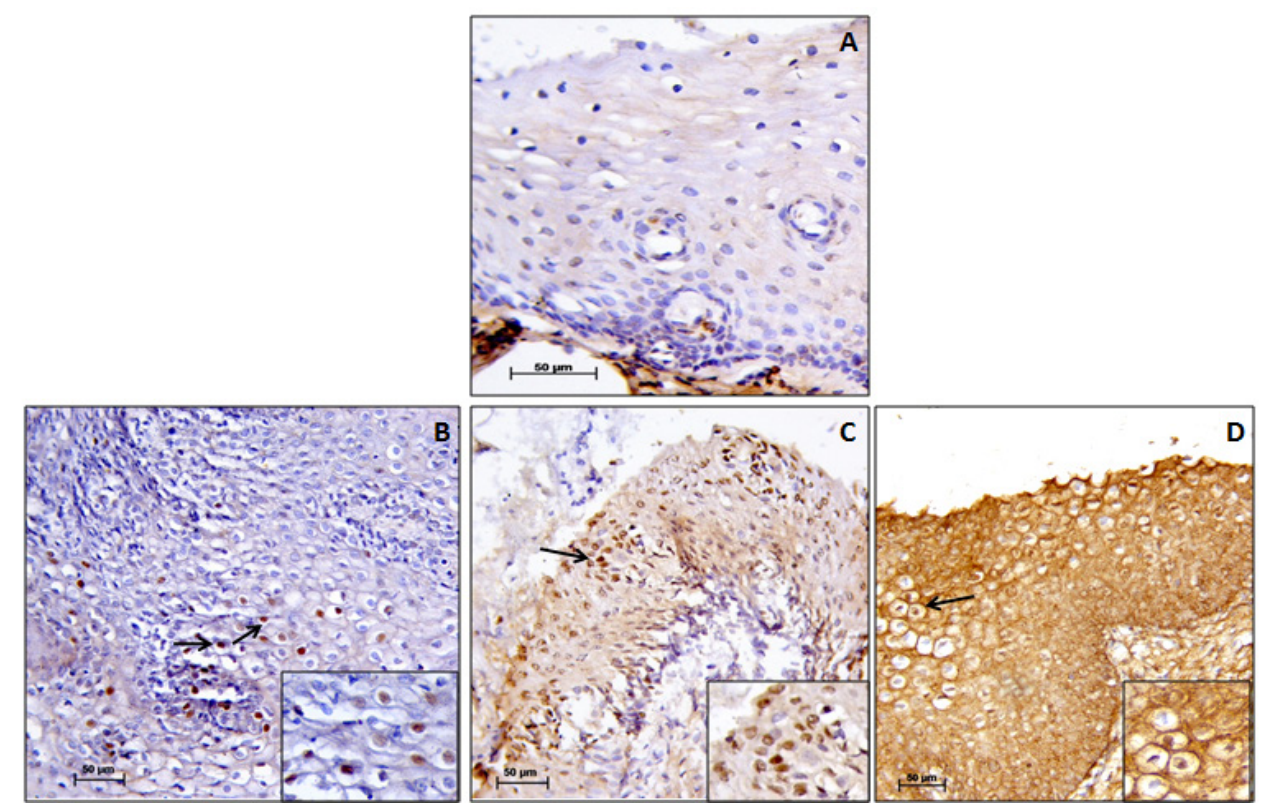

Figure 2. Immunohistochemistry of p16. (A), p16 immunonegativity (score 0); (B), rare singly dispersed p16 staining (score 1); (C), patchy but strong p16 staining (score 3); (D), strong and diffused p16 staining (score 4). The magnification of the main and insert image is $20 \mathrm{X}$ and $40 \mathrm{X}$, respectively. Scoring according to the intensity of the staining. Black arrows denote p16 immunopositive cells.

pre-neoplastic cases.

p16 overexpression has been considered as sign of integration of HPV (Chuerduangphui et al., 2018; Izadi Mood et al., 2012). IHC expression of p16 was observed only in dysplastic or neoplastic cells and was never expressed in normal cervical epithelium (Lesnikova et al., 2009) and according to many authors p16 expression appears to be a robust, specific sensitive biomarker of cervical neoplasia (Dray et al., 2005; Chuerduangphui et al., 2018). In our study p16 overexpression was highly significant and showed positive immunoreactivity in majority of the cervical pre-neoplastic lesions. Overexpression progressively increased from CIN I to CIN II but reduced in CIN III. Similar results were observed in different studies (Klaes et al., 2001; W Feng et al., 2007; Izadi Mood et al., 2012; Sarma et al., 2017) in which they also reported that there is a decreased p16

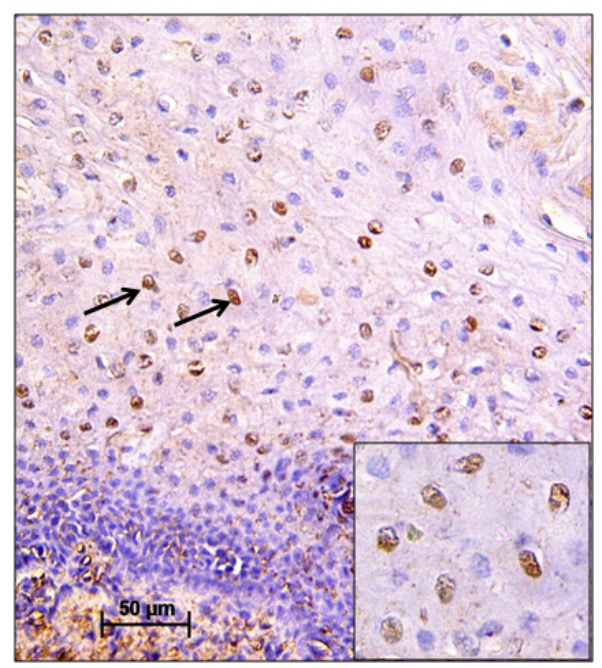

Figure 3. CIN I Showing Nuclear Immunopositivity for p53 (Black Arrows). The magnification of the main and insert image is $20 \mathrm{X}$ and $40 \mathrm{X}$, respectively. overexpression in invasive cervical cancer in comparison to high grade cervical pre-neoplastic lesions. It has been seen that overall p16 immunoexpression in cervical pre-neoplastic lesions, described as overexpression, ranges from 31 to $100 \%$ (Tsoumpou et al., 2009; Von Knebel et al., 2012; Wu et al., 2014). A study by Lesnikova et al., (2009) has seen that most cases of CIN I and large proportion of CIN II and CIN III can be expected to regress spontaneously. The rate of regression of CIN III is almost threefold in comparison to the progression of invasive carcinoma. Thus, the decreased expression of p16 in high grade cervical lesions justifies the theory of regression. On the other hand many research articles expressed view that the 16 expression increases progressively with change of histopathological grade such as CIN I has the lowest expression and CIN III has the highest (Tsoumpou et al., 2009; Wu et al., 2014; Von Knebel et al., 2012) which

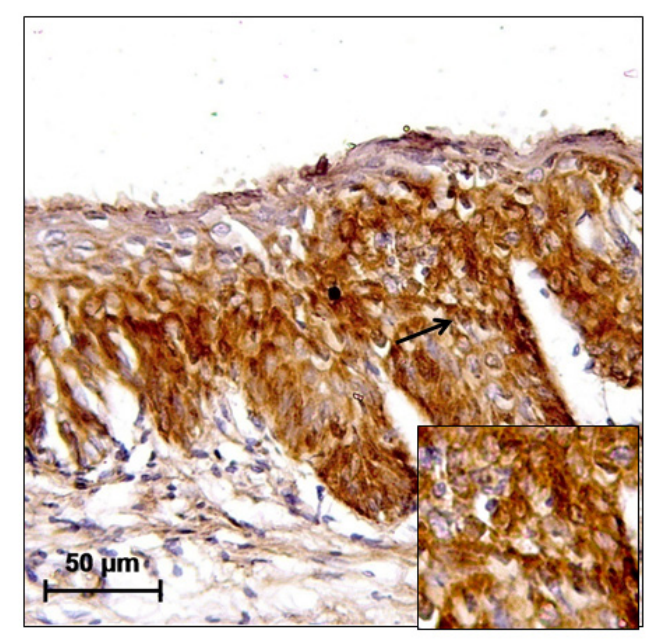

Figure 4. CIN II Showing Intense Diffuse RAR $\beta$ Immunoreactivity in Both Nuclei and Cytoplasm (Black Arrow). The magnification of the main and insert image is $20 \mathrm{X}$ and $40 \mathrm{X}$, respectively. 


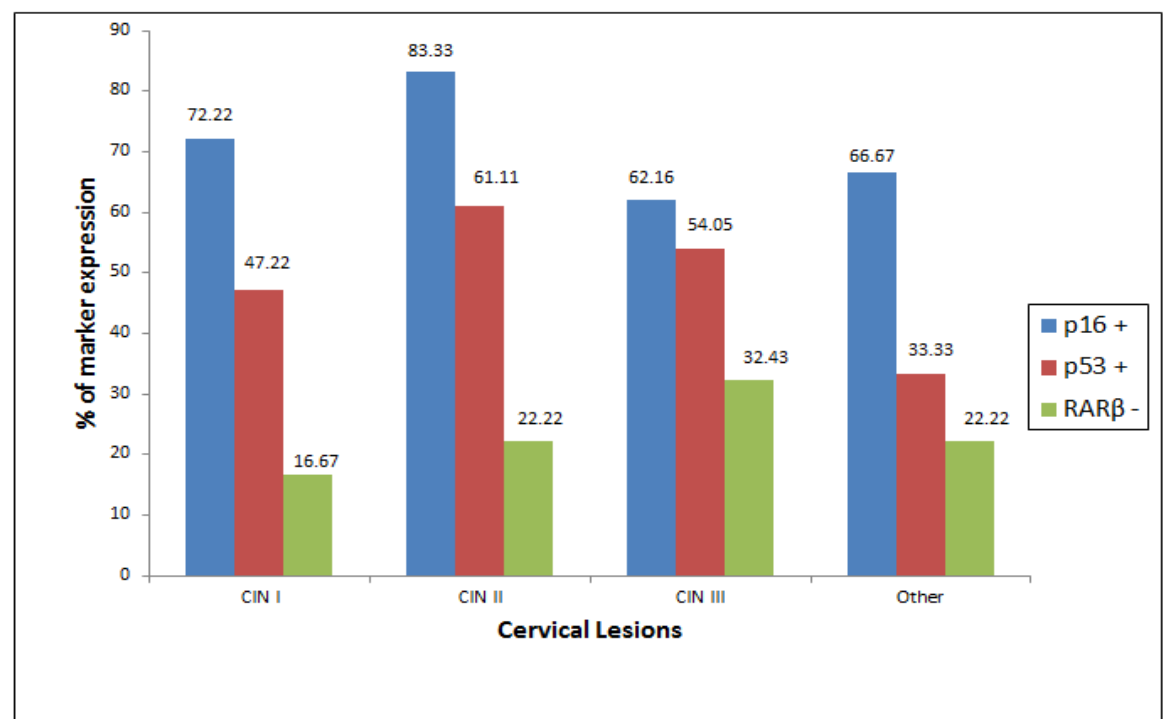

Figure 5. p16 Overexpression, p53 Immunopositivity and RAR $\beta$ Immunonegativity with Different Grades of Cervical Lesions.

subsequently progress to carcinoma cervix.

In the present study it was also found that 6 out of 9 cases of other associated lesions showed overexpression for p16, whereas many cases with histopathologically high grade lesion showed p16 negativity. Therefore, it appears that apart from $\mathrm{p} 16$ positivity of pre-neoplastic lesions, other cases such as condylomas also express p16 implying that HPV infectivity found in any of histological pattern barring CINs, are important and again shows the limitation of histopathology alone for risk prediction of cervical lesions.

p53, tumour suppressor gene, plays an important role in protection against development of cancer. However, mutation or conformational changes from suppressor to mutant p53 results in p53 oncoprotein expression (Raju et al., 2015). In our study, $51 \%$ of the cases showed positivity for p53 in cervical pre-neoplastic lesions and was significantly linked to histopathological grades CIN I to CIN II and its expression reduced in CIN III. A study by Grace et al., (2003) demonstrated that there is expression of $\mathrm{p} 53$ protein in the early stages of cervical lesions like CIN I and CIN II whereas some studies have reported that the p53 expression is a late event and was seen in advanced cervical intraepithelial lesion (CIN III) and invasive cancer (Goel et al., 2012). An Indian study by Raju et al., (2015) showed that there is high expression of p53 from LSIL to HSIL but another Indian study by Shukla et al., (2014) found that low percentage of p53 expression in CIN and a moderate expression of p53 in cervical carcinoma whereas an International study by $\mathrm{W}$ Feng et al., (2007) reported that p53 expressed in both cervical cancer and cervical dysplasia. Though there are some controversies in relation to p53 expression and histopathological grading, from the above studies it can be said that immunodetection of p53 oncoprotein in any cervical lesions, irrespective of its histopathological grade, carries significant risk of conversion and thus can be used as an important biomarker.

RAR $\beta$ exerts an inhibitory effect on expression of viral oncoproteins E6 and E7 thus decrease in RAR $\beta$ leads to the development of pre-neoplastic cervical lesions and cancer (De-Castro Arce et al., 2007). In this study, the expression of RAR $\beta$ decreased from CIN I to CIN III. Negative immunoreactivity for RAR $\beta$ was seen in $24 \%$ cases of CINs and was most common in high grade lesions (CIN III) followed by CIN II and CIN I. A study by Wongwarangkana et al. (2018) also expressed similar view and found that decrease expression of retinoic acid receptor occurs early in the development of cervical carcinoma and has been linked to CINs. Narayan et al., (2003) found that in the LSIL group, 11\% had low RAR $\beta$ expression whereas, in the HSIL group, $60 \%$ had a complete lack of RAR $\beta$ expression. Another study by Choi et al., (2007) discovered that all normal tissues highly express RAR $\beta$ protein, whereas no staining was detected in $43 \%$ of the squamous cell carcinoma. According to Ivanova et al., (2002), 40\% decrease of RAR $\beta 2$ mRNA was found in cervical squamous cell carcinoma.

In this study it has been found that out of 100 cases there are 6 cases showing p16 overexpression along with p53 positivity and RAR $\beta$ negativity. These cases along with CIN III followed by CIN II and CIN I respectively have the highest probability of malignant transformation. In the International arena similar results have been observed by Yim et al., (2005), Hwang et al., (2012), Lesnikova et al., (2009), Uyar and Rader (2014).

We also found that out 49 p53 negative cases, 31 cases were p16 positive and 11 cases were RAR $\beta$ negative, of which 10 were CIN III. Barring p53 positivity it has been reported that in high risk HPV-positive cases decrease expression of RAR $\beta$ may be an important step on the way towards malignant progression (De-Castro Arce et al 2007; Ivanova et al., 2002). Thus it is expected that high grade cases that are $\mathrm{p} 53$ negative but $\mathrm{p} 16$ positive and RAR $\beta$ negative also carry significant risk for malignant transformation.

Therefore, from the study it can be concluded that p16, p53 and RAR $\beta$ are equally important individually for risk categorization along with histopathological grading. However, it is pertinent to say that the combined marker 
study of these three markers including histopathology can be used for more accurate risk categorization and probably carries highest importance or value in categorizing risk and predicting cancer progression in cervical pre-neoplastic lesions.

In conclusion, we conclude that histopathological evaluation alone is inconclusive for predicting risk in cervical lesions. The use of biomarkers such as p16, p53, RAR $\beta$ in conjunction with histopathology could greatly improve the accuracy, precision and sensitivity of cervical screening program for risk categorization of pre-neoplastic and other lesions of cervix. These markers thus may be helpful particularly in developing countries for early risk assessment where the genetic testing of pre-neoplastic lesions as well as cancer treatment is too expensive and out of reach of most of the people in India.

\section{Limitation of the study}

Statistically the accuracy level of the study would be higher if more number of patients could be included in the study. Secondly, for further enhancement of the significance of the study, the cases needed to be followed up at a regular intervals for a longer period of time as it is one of the important steps to identify the outcome of these cases which could strongly support the predicted result and would have justified the conclusive role of these markers for risk categorization.

\section{Funding Statement}

This research received no specific grant from any funding agency in the public, commercial or not-for-profit sectors.

\section{Acknowledgements}

We like to thank friends and well wishers for their encouragement and frequent selfless help for pursuing the study. Special thanks to Dr. Gautam Mandal, Ex Head Department of Pathology, Chittaranjan National Cancer Institute, Kolkata for his help from time to time. We would also like to thank Director, CNCI for his overall support.

\section{References}

Bray F, Ferlay J, Soerjomataram I, et al (2018). Global cancer statistics 2018: GLOBOCAN estimates of incidence and mortality worldwide for 36 cancers in 185 countries. $C A$ Cancer J Clin, Published online 12 September 2018.

Chakravarti N, Mathur M, Bahadur S, Kumar Shukla N, Ralhan R (2003). Retinoic acid receptor-alpha as a prognostic indicator in oral squamous cell carcinoma. Int J Cancer, 103, 544-9.

Choi CH, Lee KM, Choi JJ, et al (2007). Hypermethylation and loss of heterozygosity of tumor suppressor genes on chromosome 3p in cervical cancer. Cancer Lett, 255, 26-33.

Chuerduangphui J, Pientong C, Swangphon P, et al (2018). Association of antibody to E2 protein of human papillomavirus and p16(INK4A) with progression of HPV-infected cervical lesions. Med Oncol, 35, 93.

De-Castro Arce J, Gockel-Krzikalla E, Rosl F (2007). Retinoic acid receptor beta silences human papillomavirus-18 oncogene expression by induction of de novo methylation and heterochromatinization of the viral control region. J Biol Chem, 282, 28520-9.

Dray M, Russell P, Dalrymple C, et al (2005). p16(INK4a) as a complementary marker of high-grade intraepithelial lesions of the uterine cervix. I: Experience with squamous lesions in 189 consecutive cervical biopsies. Pathology, 37,112-24.

Feng W, Xiao J, Zhang Z, et al (2007). Senescence and apoptosis in carcinogenesis of cervical squamous carcinoma. Mod Pathol, 20, 961-6.

Geisen C, Denk C, Kupper JH, Schwarz E (2000). Growth inhibition of cervical cancer cells by the human retinoic acid receptor beta gene. Int J Cancer, 85, 289-95.

Godoy A, Bazzo K, Moura L, Serafini E (2014). Expression Analysis of p53, Ki-67 and bcl-2 in Pre-Malignant Lesions of the Cervix. OJOG, 4, 462-9.

Goel M, Somani K, Mehrotra A, Singh U, Mehrotra R (2012). Immunohistochemical expression of cell proliferating nuclear antigen (PCNA) and $\mathrm{p} 53$ protein in cervical cancer. J Obstet Gynaecol India, 62, 557-61.

Grace VM, Shalini JV, lekha TT, Devaraj SN, Devaraj H (2003). Co-overexpression of $\mathrm{p} 53$ and bcl-2 proteins in HPV-induced squamous cell carcinoma of the uterine cervix. Gynecol Oncol, 91, 51-8.

Gutierrez J, Garcia-Villa E, Ocadiz-Delgado R ,et al (2015). Human papillomavirus type 16 E7 oncoprotein upregulates the retinoic acid receptor-beta expression in cervical cancer cell lines and K14E7 transgenic mice. Mol Cell Biochem, 408, 261-72.

Han CP, Kok LF, Wang PH, et al (2009). Scoring of p16(INK4a) immunohistochemistry based on independent nuclear staining alone can sufficiently distinguish between endocervical and endometrial adenocarcinomas in a tissue microarray study. Mod Pathol, 22, 797-806.

Harsh M (2010). Text book of pathology. New Delhi India: Jaypee, pp 726.

Hwang SJ, Shroyer KR (2012). Biomarkers of cervical dysplasia and carcinoma. $J$ Oncol, 2012, 507286.

IHC World. http://www.ihcworld.com/protocol_database.htm.

Ivanova T, Petrenko A, Gritsko T, et al (2002). Methylation and silencing of the retinoic acid receptor-beta 2 gene in cervical cancer. BMC Cancer, 2, 4.

Izadi-Mood N, Asadi K, Shojaei H, et al (2012). Potential diagnostic value of P16 expression in premalignant and malignant cervical lesions. J Res Med Sci, 17, 428-33.

Kim J, M Adel-el-Naggar D, Lee JS, et al (2001). Chromosome polysomy and histological characteristics in oral premalignant lesions. Cancer Epidemiol Biomarkers Prev, 10, 319-25.

Klaes R, Friedrich T, Spitkovsky D, et al (2001). Overexpression of p16(INK4A) as a specific marker for dysplastic and neoplastic epithelial cells of the cervix uteri. Int J Cancer, 92, 276-84.

Lesnikova I, Lidang M, Hamilton-Dutoit S, Koch J (2009). p16 as a diagnostic marker of cervical neoplasia: a tissue microarray study of 796 archival specimens. Diagn Pathol, $4,22$.

Mishra GA, Pimple SA, Shastri SS (2016). Prevention of cervix cancer in India. Oncology, 91, 1-7.

Narayan G, Arias-Pulido H, Koul S, et al (2003). Frequent promoter methylation of CDH1, DAPK, RARB, and HIC1 genes in carcinoma of cervix uteri: its relationship to clinical outcome. Mol Cancer, 2, 24.

Qin LX, Tang ZY, Ma ZC, et al (2002). P53 immunohistochemical scoring: an independent prognostic marker for patients after hepatocellular carcinoma resection. World J Gastroenterol, 8, 459-63.

Raju K, Punnayanapalya SS, Mariyappa N (2015). Significance of p53, pRb and Ki-67 markers in cervical intraepithelial 
lesion and malignancy. Biomed Res Ther, 2, 374-84.

Sarma U, Biswas I, Das A, et al (2017). p16INK4a Expression in Cervical Lesions Correlates with Histologic Grading - a Tertiary Level Medical Facility Based Retrospective Study. Asian Pac J Cancer Prev, 18, 2643-7.

Shukla S, Dass J, Pujani M (2014). p53 and bcl2 expression in malignant and premalignant lesions of uterine cervix and their correlation with human papilloma virus 16 and 18 . South Asian J Cancer, 3, 48-53.

Stiasny A, Freier CP, Kuhn C, et al (2017). The involvement of E6, p53, p16, MDM2 and Gal-3 in the clinical outcome of patients with cervical cancer. Oncol Lett, 14, 4467-76.

Tainio K, Athanasiou A, Tikkinen KAO, et al (2018). Clinical course of untreated cervical intraepithelial neoplasia grade 2 under active surveillance: systematic review and meta-analysis. $B M J, \mathbf{3 6 0}, \mathrm{k} 499$.

Tsoumpou I, Arbyn M, Kyrgiou M, et al (2009). p16(INK4a) immunostaining in cytological and histological specimens from the uterine cervix: a systematic review and meta-analysis. Cancer Treat Rev, 35, 210-20.

Uyar D, Rader J (2014). Genomics of cervical cancer and the role of human papillomavirus pathobiology. Clin Chem, 60, 144-6.

von Knebel Doeberitz M, Reuschenbach M, Schmidt D, Bergeron C (2012). Biomarkers for cervical cancer screening: the role of p16(INK4a) to highlight transforming HPV infections. Expert Rev Proteomics, 9, 149-63.

Wongwarangkana C, Wanlapakorn N, Chansaenroj J, Poovorawan Y (2018). Retinoic acid receptor beta promoter methylation and risk of cervical cancer. World $J$ Virol, 7, 1-9.

Wu J, Li XJ, Zhu W, Liu XP (2014). Detection and pathological value of papillomavirus DNA and p16(INK4A) and p53 protein expression in cervical intraepithelial neoplasia. Oncol Lett, 7, 738-44.

Yim EK, Park JS (2005). The role of HPV E6 and E7 oncoproteins in HPV-associated cervical carcinogenesis. Cancer Res Treat, 37, 319-24.

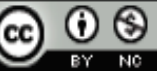

This work is licensed under a Creative Commons AttributionNon Commercial 4.0 International License. 